\title{
Evolocumab in Patients with Cardiovascular Disease
}

TO THE EDITOR: Sabatine et al. (May 4 issue) ${ }^{1}$ report the results of the Further Cardiovascular Outcomes Research with PCSK9 Inhibition in Subjects with Elevated Risk (FOURIER) trial. The addition of evolocumab to standard therapy was associated with an absolute risk reduction of 2 percentage points in both the primary and secondary end points after a median duration of follow-up of 26 months.

The fact that approximately $30 \%$ of the patients were receiving moderate-intensity statin therapy and the lack of clinical efficacy of evolocumab in the small group of patients receiving ezetimibe (presumably combined with a statin) arouse concern about the desirability of achieving such a marginal absolute risk reduction by inhibiting proprotein convertase subtilisin-kexin type 9 (PCSK9) in patients receiving high-intensity statin therapy, combined with ezetimibe, as appropriate. The results of trials that show a cardiovascular benefit of the addition of ezetimibe to statins ${ }^{2,3}$ support the role of this combination as an initial lipid-lowering strategy in patients at high cardiovascular risk.

Given the findings of the FOURIER trial, in patients at high cardiovascular risk in whom low-density lipoprotein (LDL) cholesterol goals cannot be achieved despite the use of lipid-lowering treatment, it would be more cost-effective to add evolocumab to a high-intensity statin combined with ezetimibe, as needed and if this combination has an acceptable side-effect profile. These recommendations are consistent with current guidelines that were published after PCSK9 inhibitors became available. ${ }^{4,5}$

Jaime García de Tena, M.D., Ph.D.

Hospital Universitario de Guadalajara

Guadalajara, Spain

jgtena@movistar.es

Alejandro Morales-Ortega, M.D.

Hospital Universitario de Fuenlabrada

Fuenlabrada, Spain

Gloria Pindao-Quesada, M.D.

Hospital Universitario de Guadalajara

Guadalajara, Spain

No potential conflict of interest relevant to this letter was reported.

1. Sabatine MS, Giugliano RP, Keech AC, et al. Evolocumab and clinical outcomes in patients with cardiovascular disease. $\mathrm{N}$ Engl J Med 2017;376:1713-22.

2. Cannon CP, Blazing MA, Giugliano RP, et al. Ezetimibe added to statin therapy after acute coronary syndromes. N Engl J Med 2015;372:2387-97.

3. Tsujita K, Sugiyama S, Sumida H, et al. Impact of dual lipidlowering strategy with ezetimibe and atorvastatin on coronary plaque regression in patients with percutaneous coronary intervention: the multicenter randomized controlled PRECISE-IVUS Trial. J Am Coll Cardiol 2015;66:495-507.

4. Catapano AL, Graham I, De Backer G, et al. 2016 ESC/EAS guidelines for the management of dyslipidaemias. Eur Heart J 2016;37:2999-3058.

5. Landmesser U, John Chapman M, Farnier M, et al. European Society of Cardiology/European Atherosclerosis Society Task Force consensus statement on proprotein convertase subtilisin/ kexin type 9 inhibitors: practical guidance for use in patients at very high cardiovascular risk. Eur Heart J 2016 October 27 (Epub ahead of print).

DOI: 10.1056/NEJMc1708587

TO THE EDITOR: In the FOURIER trial, Sabatine et al. found a reduction in the risk of cardiovascular events among patients with preexisting atherosclerotic disease who received evolocumab on a background of statin therapy. The data show geographic heterogeneity with respect to clinically serious secondary end points. As shown in Figure S5 in the Supplementary Appendix (available with the full text of the article at NEJM.org), a total of 17,335 patients from Europe (63\% of the enrolled population) derived a lesser benefit from evolocumab on the key secondary composite end point of cardiovascular death, myocardial infarction, or stroke (hazard ratio, 0.90; 95\% confidence interval [CI], 0.80 to 1.01) than patients from North America (hazard ratio, 0.62; $95 \%$ CI, 0.51 to 0.76 ) ( $\mathrm{P}=0.01$ for the interaction).

The inconsistency of the benefit across major regions may have considerable cost-effectiveness implications. We estimate that the number of patients who would need to be treated for 3 years to prevent one cardiovascular death, myocardial infarction, or stroke is almost 4 times as high in Europe as in North America. Under the assumption that the annual list price of evolocumab is $\$ 14,350,{ }^{1}$ preventing one such event would cost approximately $\$ 861,000$ in North America and $\$ 3,314,850$ in Europe. We wonder whether these variations were driven by baseline risk differences, a discrepancy between Europe and North America in rates of diagnosis of cardiovascular 
events (event rates in the placebo group, $6.7 \%$ vs. $10.6 \%$, or differences in adherence to treatment.

Huseyin Naci, Ph.D.

London School of Economics and Political Science

London, United Kingdom

h.naci@lse.ac.uk

Mandeep R. Mehra, M.D.

Brigham and Women's Hospital Heart and Vascular Center Boston, MA

No potential conflict of interest relevant to this letter was reported.

1. Kazi DS, Moran AE, Coxson PG, et al. Cost-effectiveness of PCSK9 inhibitor therapy in patients with heterozygous familial hypercholesterolemia or atherosclerotic cardiovascular disease. JAMA 2016;136:743-53.

DOI: 10.1056/NEJMc1708587

TO THE EDITOR: The FOURIER trial showed that evolocumab significantly reduced the risk of cardiovascular events among patients with atherosclerotic cardiovascular disease who were receiving statin therapy. The percentage of patients with diabetes in this trial (36.6\%) was higher than in most secondary-prevention trials of statins. ${ }^{1}$ It would be interesting to know the effect of evolocumab in relation to the diabetic status of the patients. Notably, in patients with or without diabetes, statins result in a similar reduction in cardiovascular risk ${ }^{1}$ and similar favorable effects on coronary atherosclerosis ${ }^{2}$ for a given magnitude of LDL cholesterol lowering. In contrast, the clinical benefit of the nonstatin agent ezetimibe is most pronounced in patients who have diabetes. ${ }^{3}$

The important insights provided by the FOURIER trial substantiate the concept that lowering the LDL cholesterol level below current targets is associated with a better clinical prognosis, but they also point to the need to identify patients who are likely to derive the greatest clinical benefit from this generally effective (yet expensive) intervention. Assessment of the clinical effects of evolocumab in patients with diabetes, who frequently have treatment-resistant dyslipidemia, complex atherosclerotic disease, and recurrent cardiovascular events, ${ }^{4}$ may enhance clinical decision making regarding the lowering of LDL cholesterol levels in very high-risk patients.

Konstantinos C. Koskinas, M.D.

Stephan Windecker, M.D.

Bern University Hospital

Bern, Switzerland

konstantinos.koskinas@insel.ch
Dr. Windecker reports receiving research contracts to his institution from Abbott, Biosensors International, Biotronik, Boston Scientific, Cordis, Medtronic, and St. Jude Medical. No other potential conflict of interest relevant to this letter was reported.

1. Cholesterol Treatment Trialists' (CTT) Collaboration. Efficacy and safety of more intensive lowering of LDL cholesterol: a meta-analysis of data from 170,000 participants in 26 randomised trials. Lancet 2010;376:1670-81.

2. Stegman B, Puri R, Cho L, et al. High-intensity statin therapy alters the natural history of diabetic coronary atherosclerosis: insights from SATURN. Diabetes Care 2014;37:3114-20.

3. Cannon CP, Blazing MA, Giugliano RP, et al. Ezetimibe added to statin therapy after acute coronary syndromes. $\mathrm{N}$ Engl J Med 2015;372:2387-97.

4. Koskinas KC, Siontis GC, Piccolo R, et al. Impact of diabetic status on outcomes after revascularization with drug-eluting stents in relation to coronary artery disease complexity: patientlevel pooled analysis of 6081 patients. Circ Cardiovasc Interv 2016;9(2):e003255.

DOI: 10.1056/NEJMc1708587

TO THE EDITOR: The FOURIER trial showed no benefit of evolocumab on cardiovascular mortality after 26 months, and there was a nonsignificant increase in deaths from any cause among patients who received evolocumab as compared with those who received placebo (444 deaths vs. 426 deaths). However, there was a 1.2-percentagepoint absolute difference in the rate of myocardial infarction in the evolocumab group. One explanation is that most of the myocardial infarctions in the trial were not ST-segment elevation myocardial infarctions (STEMIs) but were related to elevated troponin levels of unclear clinical significance. These myocardial infarctions of lesser severity could be related to the fact that more patients underwent revascularization (which is associated with elevated troponin levels) in the placebo group than in the evolocumab group. Could the authors provide rates of STEMI, non-STEMI with a risk of a Thrombolysis in Myocardial Infarction (TIMI) risk score greater than 2, and non-STEMI with a TIMI risk score of 2 or less?

Furthermore, the high price of PCSK9 therapy - $\$ 14,000$ per year ${ }^{1}$ - and the large number needed to treat must be considered. The cost to avert one myocardial infarction is $\$ 2.4$ million, and the cost to avert one stroke is $\$ 7.5$ million. The proposal by Amgen ${ }^{1}$ to issue a rebate if a myocardial infarction or stroke occurs is no real discount; since modern event rates are low, this amounts to a $5 \%$ reduction in price. Unreasonably high drug prices in the United States and globally are a major concern. 
Rita F. Redberg, M.D.

University of California, San Francisco

San Francisco, CA

Vinay Prasad, M.D., M.P.H.

Oregon Health and Science University

Portland, OR

prasad@ohsu.edu

No potential conflict of interest relevant to this letter was reported.

1. Garde D. A pricey drug cuts cardiovascular risks in clinical trial — but will insurers cover it? STAT News. March 17, 2017 (https:/ www.statnews.com/2017/03/17/amgen-repatha-cardiovascular/).

DOI: 10.1056/NEJMc1708587

TO THE EDITOR: The article by Sabatine et al. on the effects of evolocumab in patients with cardiovascular disease clearly shows that evolocumab, added to statin therapy, decreases both LDL cholesterol levels and the risk of cardiovascular events, including myocardial infarction and stroke. However, more intensive lowering of LDL cholesterol levels, as compared with less intensive therapy with statins, does not lead to a decrease in overall mortality.

This mortality paradox appears to be a reproducible phenomenon. High-intensity statin therapy in the Treating to New Targets (TNT) trial, the Incremental Decrease in Endpoints Through Aggressive Lipid Lowering (IDEAL) trial, and the Study of the Effectiveness of Additional Reductions in Cholesterol and Homocysteine (SEARCH), the combination of ezetimibe and statin therapy in the Improved Reduction of Outcomes: Vytorin Efficacy International Trial (IMPROVE-IT), and now the combination of evolocumab with a statin in the FOURIER trial all fail to reduce overall mortality, as compared with less intensive LDL cholesterol-lowering therapy that includes statins. These findings suggest that a common biologic phenomenon could be at play. ${ }^{1}$

A total of 1440 patients were treated concomitantly with ezetimibe. It would be interesting to look at the data on hospitalization for unstable angina and coronary revascularization, given the differential effect of evolocumab on the primary and key secondary outcome in this group of patients.

José P.L. Nunes, M.D.

University of Porto

Porto, Portugal

jplnunes@med.up.pt
No potential conflict of interest relevant to this letter was reported.

1. Nunes JPL. Statins and the cholesterol mortality paradox. Scott Med J 2017;62:19-23.

DOI: $10.1056 / N E J M c 1708587$

THE AUTHORS REPLY: García de Tena et al. comment on absolute risk reductions but neglect to account for treatment duration. The 2-percentagepoint absolute reduction in the rate of cardiovascular death, myocardial infarction, or stroke with evolocumab occurred over a period of 2.2 years. In comparison, it took nearly 5 years to see a 2.2-percentage-point reduction in major vascular events with high-intensity statin therapy versus moderate-intensity statin therapy. ${ }^{1}$ The number needed to treat to prevent one major vascular event over 5 years would be 45 with high-intensity statin therapy and only 30 with evolocumab. Their statement that there was no efficacy in patients receiving ezetimibe is incorrect; there was no heterogeneity in the benefit of evolocumab according to ezetimibe use.

Naci and Mehra note significant heterogeneity in the hazard ratio for the key secondary end point according to geographic region, but without correction for multiple hypothesis testing. Moreover, there was no heterogeneity for the primary end point. There were no clinically important differences in base line characteristics, adherence to treatment tended to be better in Europe, and, unsurprisingly, among the individual outcomes, revascularization rates differed most according to region.

Koskinas and Windecker inquire about the efficacy of evolocumab in patients with diabetes. These data have not been analyzed.

Redberg and Prasad speculate that the reduction in the risk of myocardial infarction could have been driven by elevated troponin levels of unclear clinical significance in patients who underwent revascularization. The data in the FOURIER trial parallel those from similar cohorts described in the literature that clearly show that such a supposition is unfounded, since type 4a myocardial infarctions (related to revascularization) accounted for only approximately $1 \%$ of all myocardial infarctions, and the majority of patients with spontaneous myocardial infarctions had troponin levels that were 10 or more times 
the upper limit of the normal range..$^{2,3}$ As noted above, the number needed to treat with evolocumab compares favorably to that with high-intensity statins, once the duration of treatment is taken into account.

Nunes comments on the lack of a benefit with respect to mortality in recent trials involving patients who were already receiving moderateintensity statin therapy. Rather than a paradox, we think this observation reflects, first, the lower case fatality rate associated with myocardial infarction and stroke than that in early trials (e.g., the Scandinavian Simvastatin Survival Study [4S]) that showed a mortality benefit and, second, the need for prolonged follow-up to see whether reductions in the risk of nonfatal myocardial infarction and stroke translate into reduced cardiovascular mortality. Genetic variants in PCSK9 that lower LDL cholesterol levels on a lifelong basis confer the same reduction in coronary mortality as do analogous variants in $\mathrm{HMGCR}^{4}$; these findings support our contention.
Marc S. Sabatine, M.D., M.P.H.

Robert P. Giugliano, M.D.

Brigham and Women's Hospital

Boston, MA

msabatine@partners.org

Terje R. Pedersen, M.D.

Oslo University Hospital

Oslo, Norway

Since publication of their article, Dr. Sabatine reports receiving honoraria from Janssen Research and Development. No further potential conflict of interest relevant to this letter was reported.

1. LaRosa JC, Grundy SM, Waters DD, et al. Intensive lipid lowering with atorvastatin in patients with stable coronary disease. N Engl J Med 2005;352:1425-35.

2. Kidd SK, Bonaca MP, Braunwald E, et al. Universal classification system type of incident myocardial infarction in patients with stable atherosclerosis: observations from Thrombin Receptor Antagonist in Secondary Prevention of Atherothrombotic Ischemic Events (TRA $2^{\circ}$ P)-TIMI 50. J Am Heart Assoc 2016;5(7): e003237.

3. Bonaca MP, Wiviott SD, Morrow DA, et al. Reduction in subtypes and sizes of myocardial infarction with ticagrelor in PEGASUS-TIMI 54. Circulation 2015;132:Suppl 3:A11594. abstract. 4. Ference BA, Robinson JG, Brook RD, et al. Variation in PCSK9 and HMGCR and risk of cardiovascular disease and diabetes. N Engl J Med 2016;375:2144-53.

DOI: 10.1056/NEJMc1708587

\section{Trial of Minocycline in Clinically Isolated Syndrome of Multiple Sclerosis}

TO THE EDITOR: The conclusion of Metz et al. (June 1 issue) $^{1}$ - that the risk of conversion from a clinically isolated syndrome to multiple sclerosis was significantly lower with minocycline than with placebo at 6 months - may not be entirely well founded. The baseline imbalance between the two randomly assigned groups may have penalized the placebo group by including a larger proportion of patients with spinal cord and multifocal symptoms at onset, a larger proportion of patients with two or more enhancing lesions on magnetic resonance imaging, and a larger number of patients fulfilling the 2010 McDonald criteria ${ }^{2}$ of multiple sclerosis. The results are inconsistent with other studies such as the RECYCLINE trial. ${ }^{3}$ A recent Cochrane review ${ }^{4}$ showed that early treatment with nearly all disease-modifying drugs reduced the probability of short-term conversion to multiple sclerosis, although the clinical benefit with respect to long-term relapse and disability worsening remains uncertain.

\author{
Maria Donata Benedetti, M.D., Ph.D. \\ Verona University Hospital \\ Verona, Italy \\ mariadonata.benedetti@ospedaleuniverona.it \\ Graziella Filippini, M.D. \\ Alessandra Solari, M.D. \\ Istituto Neurologico Carlo Besta \\ Milan, Italy
}

Dr. Solari reports having been a board member of Merck Serono and Novartis and having received speaker's fees from Almirall, Excemed, Merck Serono, Sanofi Genzyme, and Teva Pharmaceutical Industries. No other potential conflict of interest relevant to this letter was reported.

1. Metz LM, Li DKB, Traboulsee AL, et al. Trial of minocycline in a clinically isolated syndrome of multiple sclerosis. $\mathrm{N} \mathrm{Engl} \mathrm{J}$ Med 2017;376:2122-33.

2. Polman CH, Reingold SC, Banwell B, et al. Diagnostic criteria for multiple sclerosis: 2010 revisions to the McDonald criteria. Ann Neurol 2011;69:292-302.

3. Sørensen PS, Sellebjerg F, Lycke J, et al. Minocycline added to subcutaneous interferon $\beta$-1a in multiple sclerosis: randomized RECYCLINE study. Eur J Neurol 2016;23:861-70.

4. Filippini G, Del Giovane C, Clerico M, et al. Treatment with disease-modifying drugs for people with a first clinical attack 